Editorial

\section{"Patients' choice"}

\section{Rossor}

$\mathrm{P}$ atients are becoming better informed and are increasingly involved in complex decisions of management. Patients are also becoming more involved in setting the medical research agenda. We know many major advances in medical science emerge from research that had no obvious clinical application; nevertheless, input into the direction of translational research is important. Equally it would be useful to have an input into the product of research-that is, publication. Bibliometrics reflect the interest and value to researchers themselves and are powerful drivers of further funding. A bibliometric that incorporates patient views, and we are all patients at some point, remains elusive. However, the journal would welcome patients' views on our publications and to that end we are introducing "patients' choice". We already have an editor's choice-a paper considered to be of particular interest or importance-that is freely downloadable from the website. The "patients' choice" will also be a free download. It will be an article thought to be of particular interest to patients and we have engaged the assistance of the $B M J$ Patient Forum to identify such articles. The decision to publish remains with the editors-like the editor's choice, the patients' choice is only made after the issue contents have been decided. We hope that authors will welcome this recognition of their papers and a wider readership will find it useful.

J Neurol Neurosurg Psychiatry 2005;76:1185

\title{
White matter hyperintensities: a target for the prevention of cognitive decline?
}

\section{Leys, S Bombois}

\section{Lowering blood pressure might decrease the rate of cognitive decline in $\epsilon 4$ carriers and in subjects with white matter hyperintensities, a risk factor for cognitive decline}

W hite matter hyperintensities (WMH) are frequent in apparently normal elderly subjects, in patients with vascular risk factors such as high blood pressure levels, in stroke patients, and in patients with either vascular dementia or Alzheimer's disease $(A D)$. In this issue, the papers by Garde et al (see pages 1289-91), Jokinen et al (see pages 1229-33), ${ }^{2}$ and de Leeuw et al (see pages 1286-8 $)^{3}$ provide evidence that WMH also influence cognitive functions independently of the underlying pathology. These three studies provide new information leading to potential strategies to prevent cognitive decline.

The more severe the progression of WMH over time, the more severe the cognitive decline in both $\mathrm{AD}$ patients ${ }^{3}$ and normal elderly subjects. ${ }^{1}$ This finding suggests that WMH should be regarded as risk factors for cognitive decline per se. Therefore, any therapeutic option that may slow the progression of WMH might help decrease the rate of cognitive decline in $\mathrm{AD}$ patients ${ }^{3}$ and normal subjects. ${ }^{1}$ As the major risk factor for WMH is arterial hypertension, blood pressure lowering drugs should therefore be tested first to examine this hypothesis in patients who already have WMH. When the role of cerebral atrophy is taken into account, the influence of WMH on cognitive functions remains significant, ${ }^{2}$ suggesting that the results of previous studies ${ }^{1-3}$ are not the consequence of cerebral atrophy which is frequently associated with WMH. After a stroke, WMH are also independently associated with cognitive impairment. ${ }^{3}$ The hypothesis of de Leeuw et al, ${ }^{3}$ that blood pressure lowering in patients with WMH might decrease the rate of cognitive decline in $\mathrm{AD}$ patients, is therefore valid also for stroke patients. However, white matter changes on CT scan in stroke patients are associated with an increased risk of stroke recurrence. ${ }^{4}$ It is, therefore, difficult to evaluate the effect of blood pressure lowering drugs on WMH progression only: the reduction in stroke recurrences may account for some of the results, besides a possible reduction in the rate of progression of WMH.
The aim of the three studies ${ }^{1-3}$ was not to explain why only some patients have rapid progression of WMH over time. Genetic factors might explain differences in the susceptibility of cerebral white matter to high blood pressure: in the Rotterdam study ${ }^{5}$ : (i) APOE $\in 4$ carriers had significantly more subcortical WMH than APOE $€ 3 \in 3$ carriers, irrespective of their baseline levels of blood pressure; ${ }^{5}$ and (ii) an $€ 4$ allele and a high level of blood pressure were strongly associated with the presence of WMH, while hypertension alone or the presence of an $\in 4$ allele alone were not. ${ }^{5}$

These radiological ${ }^{1-3}$ and genetic ${ }^{5}$ findings open a window for selective prevention of cognitive impairment in high risk subjects. The hypothesis that lowering blood pressure might be more beneficial in subjects with WMH and in $€ 4$ carriers should now be tested in randomised trials.

\section{ACKNOWLEDGEMENTS}

The authors thank Professor Florence Pasquier for her critical reading of a preliminary version of this editorial commentary.

J Neurol Neurosurg Psychiatry 2005;76:1185-1186.

doi: 10.1136/jnnp.2005.064766

\section{Authors' affiliations}

D Leys, Department of Neurology, Stroke, EA2691, Lille University Hospital, Lille, France S Bombois, Department of Neurology, Memory Units, EA2691, Lille University Hospital, Lille, France

Correspondence to: D Leys, Department of Neurology (EA2691), Lille University Hospital, F-59037 Lille, France; dleys@chru-lille.fr 


\section{REFERENCES}

1 Garde E, Mortensen EL, Rostrup E, et al. Decline in intelligence is associated with progression in white matter hyperintensity volume. J Neurol Neurosurg Psychiatry 2005;76:1289-91.

2 Jokinen $H$, Kalska $H$, Mäntylä $R$, et al.

White matter hyperintensities as a predictor of neuropsychological deficits post stroke. J Neurol Neurosurg Psychiatry 2005;76: 1229-33.

3 de Leeuw F-E, Barkhof F, Scheltens Ph. Progression of cerebral white matter lesions in Alzheimer's disease: a new window for therapy? I Neurol Neurosurg Psychiatry 2005;76:1286-8.

\author{
4 Hénon $\mathrm{H}$, Vroylandt $\mathrm{P}$, Daems $\mathrm{C}$, et al \\ Leukoaraiosis more than dementia is a \\ predictor of stroke recurrence. Stroke \\ 2003:34:2935-40. \\ 5 de Leeuw FE, Richard F, de Groot JC, et al. \\ Interaction between hypertension, apoE, and \\ cerebral white matter lesions. Stroke
} 2004;35:1057-60 turned on, though again in the offdrug condition. We have recently shown that a significant difference in dopamine transporter capacity, though not in dopamine uptake, can be seen between pre-STN stimulation and postSTN insertion but with stimulation turned off. ${ }^{4}$ Thus electrode placement resulting in a "microsubthalamotomy" could have an effect on its own.

Nevertheless STN stimulation is like other traditional functional neurosurgical ablative techniques in producing symptomatic relief without affecting the biology of the disease.

What is the cause of the discrepancy between the experimental findings and this clinical study that rules out neuroprotective effects? The experimental papers used an artificial model of Parkinson's disease-that is, the 6OHDA model which does not reproduce all the features of the disease. Furthermore in that model, STN ablation with kainic acid is used instead of STN stimulation to show the potential neuroprotective effects of STN suppression on the substantia nigra.

The paper by Hilker et al also raises questions about the focus of future neurosurgical approaches in Parkinson's disease. The investigators point out quite correctly that STN stimulation, which can be carried out bilaterally, has been found to be beneficial in numerous retrospective studies. However, it clearly only provides symptomatic benefit and therefore falls into the realm of traditional functional neurosurgical approaches such as STN ablation. In this context it is worthwhile asking whether the current prospective randomised controlled trial (the PD
Surg trial) comparing bilateral STN stimulation with the best medical treatment is a wise investment of money. Very likely the study is going to prove the obvious. New attempts have been made by several neurosurgical groups to modify disease biology in Parkinson's disease and by doing so to achieve actual neuroprotection. These include attempts at the direct infusion of glial cell line derived neurotrophic factor (GDNF) using convection enhanced delivery, which seems to have long term effects in the same range as STN stimulation and looks very promising. ${ }^{56}$ In addition, while still in the experimental phase the elegant approach of converting the excitatory glutamatergic STN by means of gene therapy into an inhibitory GABAergic nucleus - which is currently being tested in patients after successful results in an experimental Parkinson's model—also looks promising. $^{78}$

Besides the worthwhile clinical information gained from Hilker's paper, another of its merits is to point out that, while STN stimulation is highly effective and beneficial in patients with Parkinson's disease, we should be looking for more sophisticated means of attacking the disease biology and hopefully modifying it.

J Neurol Neurosurg Psychiatry 2005;76:1186-1187.

doi: 10.1136/jnnp.2004.061481

Correspondence to: Professor Peter C Warnke, The University of Liverpool, Department of Neuroscience, The Walton Centre for Neurology and Neurosurgery, Lower Lane, Liverpool L97ป, UK; p.c.warnke@liv.ac.uk

Competing interests: none declared

\section{REFERENCES}

1 Benazzous A, Paillat B, Ni ZG, et al. Implication of the subthalamic nucleus in the pathophysiology and pathogenesis of Parkinson's disease. Cell Transplant 2000;9:215-21.

2 Piallat B, Benazzouz A, Benabid AL. Subthalamic nucleus lesion in rats prevents dopaminergic nigral neuron degeneration after striatal 6-OHDA injection: behavioural and immunohistochemical studies. Eur J Neurosci 1996;8:1408-14.

3 Nakao N, Nakai E, Nakai K, et al. Ablation of the subthalamic nucleus supports the survival of nigral dopaminergic neurons after nigrostriatal lesions induced by the mitochondrial toxin 3nitropropionic acid. Ann Neurol 1999;45:640-51

4 Warnke PC, Fox S, Tyne $H$, et al. Selective effect of bilateral STN-electrode insertion and 
STN-stimulation on dopamine transporter binding and glucose utilisation. Acta Neurochir 2004; 146:870

5 Gill SS, Patel NK, Hotton GR, et al. Direct brain infusion of glial cell line-derived neurotrophic factor in Parkinson disease. Nat Med 2003;9:589-95. Epub 2003 Mar 31.
6 Patel NK, Bunnage $M$, Plaha $P$, et al. Intraputamenal infusion of glial cell line-derived neurotrophic factor in PD; a two-year outcome study. Ann Neurol 2005;57:298-302.

7 Luo J, Kaplitt MG, Fitzsimons HL, et al. Subthalamic GAD gene therapy in a
Parkinson's disease rat model. Science 2002;298:425-9.

8 During MJ, Kaplitt MG, Stern MB, et al. Subthalamic GAD gene transfer in Parkinson disease patients which are candidates for deep brain stimulation. Hum Gene Ther 2001;12:1589-9.

\section{DWI and acute stroke prognosis: a simple approach}

\section{Di Piero}

\section{Analysis of DWI lesion patterns may identify stroke patients at high risk of complications}

n the paper by Bang et al in this issue (see pages $1222-8$ ), the authors deal with the possibility of predicting clinical outcome in stroke patients by considering the diffusion weighted imaging (DWI) lesion patterns. This is a new approach to MRI findings which gives more emphasis to the location of DWI alterations than to their volume or number.

Although thrombolysis is the most effective treatment for acute stroke, it is still limited to a few patients because of its strict temporal therapeutic window. It follows that most therapeutic efforts are devoted to controlling the neurologic and medical complications. Therefore, early identification of patients at high risk of complications could potentially help to differentiate acute stroke therapies by selecting those patients who should be monitored and treated more aggressively. Early identification could also thus help to allocate medical resources better.

A simple evaluation of the DWI lesion pattern showed that patients presenting with internal borderzone infarcts have a more unstable hospital course. This subgroup of stroke patients may have a particular pathophysiological condition, probably linked to a precarious haemodynamic state associated with the occurrence of large artery disease.

If these internal borderzone infarcts are located in the supraventricular and paraventricular areas, we might hypothesise an unstable penumbral state. This should mainly involve the white matter which has been recently shown to present areas of potentially salvageable tissue as well as similar resistance to ischaemia of grey matter. ${ }^{1}$ In addition, a PET comparative study has shown that a DWI lesion may contain not only tissue destined for infarction but also penumbral areas that may still be saved. ${ }^{2}$

Another major early neurological complication is stroke recurrence, with a fatal outcome in about one fourth of cases. Kang et al demonstrated by serial DWI studies that "radiological" recurrences occurred in about one third of patients in the acute phase of stroke. ${ }^{3}$ In other words, stroke appears to be a "dynamic" phenomenon not limited to a single event but the result of multiple local or distant ischaemic insults. In their study, recurrences occurred mainly in patients with large vessel atherosclerosis and were frequently clinically silent. Looking at clinically manifested recurrences, Bang et al have showed that a DWI alterations pattern of small cortical infarcts suggested a higher risk of recurrent strokes.

Recent sophisticated imaging studies have indicated the possibility of closer insights into the pathophysiology of acute ischaemic stroke and its relationship with clinical features. However, such studies frequently require dedicated devices and research teams. A simple approach such as analysis of DWI lesion patterns might provide additional useful criteria to identify a subgroup of patients at high risk of neurological complications who should be strictly monitored. If these observations are confirmed, they may provide new opportunities for a better definition of individual therapeutic interventions in the acute phase of ischaemic stroke.

J Neurol Neurosurg Psychiatry 2005;76:1187. doi: 10.1136/jnnp.2005.064436

Correspondence to: $\mathrm{V}$ Di Piero, Department of Neurology, viale dell'Università 30 , Rome 00185, ltaly; vittorio.dipiero@uniromal.it

\section{REFERENCES}

1 Falcao AL, Reutens DC, Markus R, et al. The resistance to ischemia of white and gray matter after stroke. Ann Neurol 2004;56(5):695-701.

2 Guadagno JV, Warburton EA, Aigbirhio FI, et al. Does the acute diffusion-weighted imaging lesion represent penumbra as well as core? A combined quantitative PET/MRI voxel-based study. J Cereb Blood Flow Metab 2004;24(11): 1249-54.

3 Kang DW, Latour LL, Chalela JA, et al. Early and late recurrence of ischemic lesion on MRI: evidence for a prolonged stroke-prone state? Neurology 2004;63(12):2261-5. 